\title{
MODULAR PERMUTATION REPRESENTATIONS $\left({ }^{1}\right)$
}

\author{
BY
}

\section{L. SCOTT}

ABSTRACT. A modular theory for permutation representations and their centralizer rings is presented, analogous in several respects to the classical work of Brauer on group algebras.

Some principal ingredients of the theory are characters of indecomposable components of the permutation module over a $p$-adic ring, modular characters of the centralizer ring, and the action of normalizers of $p$-subgroups $P$ on the fixed points of $P$. A detailed summary appears in [15].

A main consequence of the theory is simplification of the problem of computing the ordinary character table of a given centralizer ring. Also, some pre viously unsuspected properties of permutation characters emerge. Finally, the theory provides new insight into the relation of Brauer's theory of blocks to Green's work on indecomposable modules.

The purpose of this article is to present proofs of the results announced in [15]. Statements of these results have been included here, though a number of explanatory remarks and general background references have not been repeated.

With the exception of $\$ 0$, the sections of this paper have been named according to the features of the classical modular theory with which they are most closely related.

0 . The centralizer ring. Throughout this paper $G$ is a finite group acting on a finite set $\Omega$ (perhaps not transitively or faithfully) and $p$ is a fixed prime.

If $S$ is any commutative ring with identity, we define the $S$-centralizer ring $V_{S}(G)=V_{S}(G, \Omega)$ to be the collection of all matrices with entries from $S$ that commute with the permutation matrices determined (with respect to some fixed ordering of $\Omega$ ) by elements of $G$. In case $S$ is the ring of rational integers, we write only $V(G)$ for $V_{S}(G)$ and refer to $V(G)$ as the centralizer ring. The standard basis matrices $\left\{A_{i}\right\}_{i=1}^{r}$ are obtained from the full set $\left\{O_{i}\right\}_{i=1}^{r}$ of orbits of $G$ on $\Omega \times \Omega$ by setting the $\alpha, \beta$ entry of $A_{i}$ equal to 1 for $(\alpha, \beta) \in O_{i}$ and 0 otherwise. These matrices always form an $S$-basis for $V_{S}(G)$. In particular, $V_{S}(G)$ is isomorphic to the tensor product $S V(G)$.

Received by the editors August 27, 1971.

AMS (MOS) subject classifications (1970). Primary 20C20; Secondary 16A26, 20 C05.

Key words and phrases. Indecomposable module, vertex, centralizer ring, decomposition numbers, Brauer homomorphism, Brauer's first fundamental theorem, Green corre spondence, Brauer's seconds fundamental theorem, defect group, defect 0 and 1 , splitting field.

(1) Manuscript preparation supported by NSF-GP-9572. 
The notation $S \Omega$ refers to the usual $S$-free $S G$ module obtained from the action of $G$ on $\Omega$; we regard $S \Omega$ also as a $V_{S}(G)$ module in the obvious way. (2)

In this paper $S$ will usually be $K, R$, or $F$ where $K$ is a $p$-adic number field, $R$ is the ring of local integers in $K$, and $F$ is the residue class field $R / \pi R . \pi$ is a generator of the maximal ideal of $R$. We use the notation $\bar{x}$ for the image of $x$ under some (hopefully obvious) map $X \rightarrow X / \pi X$ of an $R$-module $X$ containing $x$.

$$
\widetilde{V_{R}(G)} \cong V_{F}(G) \text {. }
$$

Proof. This is an immediate consequence of the isomorphism mentioned in the first paragraph.

(0.2) Proposition 1. Let $M, N$ be $R G$-indecomposable components of $R \Omega$. Then

(a) $\overline{\operatorname{Hom}}_{R G}(M, N) \cong \operatorname{Hom}_{F G}(\bar{M}, \bar{N})$.

(b) $\bar{M}$ is indecomposable and has the same vertex as $M$.

Proof. Of course the natural map $\overline{\operatorname{Hom}_{R G}(M, N)} \rightarrow \operatorname{Hom}_{F G}(\bar{M}, \bar{N})$ is a monomorphism. Since the functor Hom is additive and $\overline{V_{R}(G)} \cong V_{F}(G)$, the map must be an isomorphism.

Now $\operatorname{Hom}_{R G}(M, M)$ is a completely primary ring, as is well known. Obviously, the isomorphism of $\operatorname{Hom}_{R G}(M, M)$ with $\operatorname{Hom}_{F G}(\bar{M}, \bar{M})$ is a ring homomorphism; therefore $\operatorname{Hom}_{F G}(\bar{M}, \bar{M})$ contains only one idempotent-that is, $\bar{M}$ is indecomposable.

If $P$ is a subgroup of $G$, then we deduce easily from the above ring isomorphism and D. Higman's criterion [10, Theorem 1] that $M$ is $P$-projective if and only if $\bar{M}$ is $P$-projective. So by definition (see Green $[7,1.2]$ ) $M$ has vertex $P$ if and only if $\bar{M}$ has vertex $P$.

Because of Proposition 1, many results we obtain for $V_{F}(G)$ contain implicit analogues for $V_{R}(G)$.

1. Decomposition numbers. Let $A$ be an $R$ algebra (finitely generated as an $R$ module) and $M$ an $R$-free $A$ module such that $K M$ is completely reducible. Let $B$ be an $R$ algebra acting faithfully on $M$ and inducing $\operatorname{End}_{A}(M)$ by its action.

We are interested in the case $A=R G, M=R \Omega, B=V_{R}(G, \Omega)$. However, the results in this section are purely formal and are accordingly given a more general treatment.

The following result is well known. The notation $X \mid Y$ means $X$ is isomorphic to a direct summand of $Y$.

(2) All "modules" are, by convention, finitely generated and acted upon on the right, with the exception that the action of a base ring such as $S$ is written on the left. 
(1.1) Let $e, f$ be idempotents in $B$. Then the following statements are equivalent:

(a) $e=x f y$ for some $x, y \in B$.

(b) Me $\mid M f$ (considered as $A$ modules).

(c) $e B \mid f B$ (considered as $B$ modules).

Accordingly we say that $e$ and $f$ are equivalent $(e \sim f)$ if $e=x f y$ and $f=$ zew for some $x, y, z, w \in B$. When $e, f$ are primitive then $e \sim f$ whenever one of the conditions (a), (b), (c) is satisfied.

Of course the preceding discussion is valid also for $K A, K M, K B$ (and $\bar{A}$, $\bar{M}, \bar{B}$ if $\bar{B}$ induces $\operatorname{End} \bar{A}(\bar{M})$ ).

By using equivalence classes of primitive idempotents as intermediaries we establish 1 -1 correspondences $\left[M_{j}\right] \leftrightarrow\left[U_{j}\right]$ and $\left[X_{s}\right] \leftrightarrow\left[Z_{s}\right]$ between isomorphism classes of $A$-indecomposable components of $M$ and projective indecomposable $B$ modules, and between isomorphism classes of irreducible $K A$ submodules of $K M$ and irreducible $K B$ modules.

Also there is a well-known 1-1 correspondence $\left[U_{j}\right] \leftrightarrow\left[L_{j}\right]$ between isomor phism classes of projective indecomposable $B$ modules and irreducible $\bar{B}$ modules ( $L_{j}$ is the unique irreducible quotient module of $\bar{U}_{j}$ ).

In view of these correspondences there are three kinds of "decomposition numbers" we can define:

(1) Let $Z_{s}^{0}$ be an $R$-free $B$ module with $K Z_{s}^{0} \approx Z_{s}$. Then we list the composition factors of $\bar{Z}_{s}^{0}$, writing $\bar{Z}_{s}^{0} \leftrightarrow \Sigma_{j} \tilde{d}_{s j} L_{j}$.

(2) Set $K U_{j} \approx \Sigma_{s} d_{s j} Z_{s}$.

(3) Set $K M_{j} \approx \Sigma_{s} d_{s j}^{M} X_{s}$.

(1.2) Theorem 1. Assume $K, F$ are splitting fields for $K B, \bar{B}$ respectively. Then $\tilde{d}_{s j}=d_{s j}=d_{s j}^{M}$ for all $s, j$. Also $M \approx \bigoplus \Sigma_{j}\left(\operatorname{dim}_{F} L_{j}\right) M_{j}$.

Note that the last assertion is a modular-theoretic version of the familiar $K M \approx \Sigma_{s}\left(\operatorname{dim}_{K} Z_{s}\right) X_{s}$.

Proof. The equality $\tilde{d}_{s j}=d_{s j}$ is well known [2, IX, 8]. Let $u \in B$ be a primitive idempotent with $M u \approx M_{j}$. Now the following two assertions are easily verified:

(i) For each nonnegative integer $d, d Z_{s} \mid u K B$ if and only if there exists a set of $d$ orthogonal primitive idempotents in $u K B u$ each equivalent to an idempotent $f$ with $f K B \approx Z_{s}$.

(ii) For each nonnegative integer $d, d X_{s} \mid K M u$ if and only if there exists a set of $d$ orthogonal primitive idempotents in $u K B u$ each equivalent to an idempotent $f$ with $K M f \approx X_{s}$. By definition $f K B \approx Z_{s}$ if and only if $K M f \approx X_{s}$. Thus $d_{s j}=d_{s j}^{M}$. 
To finish the proof we make two further observations:

(iii) For each nonnegative integer $l, l M_{j} \mid M$ if and only if there exists a set of $l$ orthogonal primitive idempotents in $B$ each equivalent to $u$.

(iv) The multiplicity of $\bar{U}_{j}$ as a component of $\bar{B}$ is $\operatorname{dim}_{F} L_{j}$ (see the argument on p. 419 of [4]).

The final assertion of the theorem is an obvious consequence.

The following proposition clarifies the hypothesis of Theorem 1.

(1.3) End ${ }_{K B}\left(Z_{s}\right)$, End $\bar{B}\left(L_{j}\right)$ are anti-isomorpbic to End $_{K A}\left(X_{s}\right)$, $\operatorname{End}_{A}\left(M_{j}\right) / \operatorname{Rad}\left(\operatorname{End}_{A}\left(M_{j}\right)\right)$ respectively.

In particular $K$ is a splitting field for $K B$ if and only if each $X_{s}$ is absolutely irreducible, and $F$ is a splitting field for $\bar{B}$ if and only if each $M_{j}$ is absolutely. indecomposable.

Proof. Let $e \in B$ be a primitive idempotent. Then of course $e B e \approx \operatorname{End}_{A}(M e)$. But also the left multiplications by members of $e B e$ form $\operatorname{End}_{B}(e B)$. Hence End $_{A}(M e)$ is anti-isomorphic to $\operatorname{End}_{B}(e B)$.

Since $e B$ is projective, $\operatorname{End}_{B}(e B / e \operatorname{Rad}(B))$ is a homomorphic image of End $_{B}(e B)$. Since the latter is completely primary,

$$
\operatorname{End}_{B}(e B) / \operatorname{Rad}\left(\operatorname{End}_{B}(e B)\right) \approx \operatorname{End}_{B}(e B / e \operatorname{Rad}(B)) .
$$

This proves the assertion regarding $\operatorname{End}_{\bar{B}}\left(L_{j}\right)$; the statement regarding End $_{K B}\left(Z_{s}\right)$ is established by the first paragraph of the same argument.

2. Defect groups. Let $A_{i}$ be a standard basis matrix. We define "the" defect group $D_{i}$ of $A_{i}$ to be a Sylow $p$-subgroup of $G_{\alpha \beta}$ where $(\alpha, \beta) \in O_{i}$. (Defect groups are well defined only up to conjugation by elements of $G$.).

Set $A_{i} A_{j}=\Sigma_{k} a_{i j k} A_{k}$ (in $\left.V(G)\right)$. The quantities $a_{i j k}$ are the "intersection numbers" defined by D. Higman [11]. We have

$$
a_{i j k}=\left|O_{i}(\alpha) \cap O_{j}^{*}(\beta)\right| \text { where }(\alpha, \beta) \in O_{k} \text {. }
$$

Here $O_{i}(\alpha)=\left\{\beta \mid(\alpha, \beta) \in O_{i}\right\}$ and $O_{j}^{*}=O_{j^{*}}=\left\{(\beta, \alpha) \mid(\alpha, \beta) \in O_{j}\right\}$. The proof of (2.1) is a direct matrix calculation from the definition of the $A_{i}$ 's.

(2.2) If $a_{i j k} \equiv O(p)$ then $D_{k} \leq_{G} D_{i}$ and $D_{k} \leq_{G} D_{j}$. (The notation " $\leq_{G}$ " means " $\leq$ a G-conjugate of.")

Proof. $G_{\alpha \beta}$ acts on $O_{i}(\alpha) \cap O_{j}^{*}(\beta)$ and hence so does (a suitable) $D_{k}$. If $\left|O_{i}(\alpha) \cap O_{j}^{*}(\beta)\right| \not \equiv O(p)$ then $D_{k}$ must fix a letter $\gamma \in O_{i}(\alpha) \cap O_{j}^{*}(\beta)$. Thus $D_{k}$ fixes $(\alpha, \gamma) \in O_{i}$ and $(\gamma, \beta) \in O_{j}$. The result now follows from Sylow's theorem.

For any $p$-subgroup $P$ of $G$ we define $I_{F}(P)=I_{F}(P ; G, \Omega)$ to be the set of F-linear combinations of $A_{i}$ 's satisfying $D_{i} \leq{ }_{G} P$.

Lemma 1. For $P, Q$, p-subgroups of $G$,

$$
I_{F}(P) I_{F}(Q) \leq \sum_{D_{i} \leq_{G} P_{i} D_{i} \leq_{G} Q} I_{F}\left(D_{i}\right) .
$$


Proof. Immediate from (2.2).

Now, given a primitive idempotent $e \in V_{F}$, there is an index $i$ such that $e \epsilon$ $I_{F}\left(D_{i}\right)$ and $D_{i} \leq_{G} P$ whenever $e \in I_{F}(P)$. We set $D(e)={ }_{G} D_{i}$ and call $D(e)$ "the" defect group of $e$. (See Green's proof $[7,3.3 \mathrm{~b}]$ for a discussion of how Lemma 1 guarantees the legitimacy of our definition of defect group.).

$D(e)$ is also the defect group of any idempotent equivalent to $e$. This is easily verified directly, or may be seen as a consequence of the next result.

Proposition 2. $D(e)$ is the vertex of F $R$.

To prove Proposition 2 we need an alternate description of the ideals $I_{F}(P)$. Define $N_{G, H}(x)$, for $H \leq G$ and $x \in V_{F}(H, \Omega)$, to be $\Sigma x^{g}$ where $g$ ranges over a set of right coset representatives of $H$ in $G$ (here $x^{g} \in V_{F}\left(H^{g}, \Omega\right)$ is defined in the obvious way).(3) Clearly $N_{G, H}(x) \in V_{F}(G, \Omega)$.

(2.4) $I_{F}(P ; G, \Omega)=N_{G, P}\left(V_{F}(P, \Omega)\right)$.

Proof. For $\alpha, \beta \in \Omega$ let $e_{a, \beta}$ denote the $|\Omega| \times|\Omega|$ matrix with 1 in the $\alpha, \beta$ position and 0 everywhere else. Obviously, $e_{\alpha \beta} \in V_{F}(H, \Omega)$ whenever $H \leq G_{a \beta}$. It is easy to calculate that, for $(\alpha, \beta) \in O_{i}$ and $D_{i} \leq G_{\alpha \beta}, A_{i}=N_{G, D_{i}}(x)$ where $x=\left[G_{\alpha \beta}: D_{i}\right]^{-1} e_{\alpha \beta}$. Thus if $D_{i} \leq P$ we have $A_{i}=N_{G, P}\left(N_{P, D_{i}}(x)\right) \epsilon$ $N_{G, P}\left(V_{F}(P, \Omega)\right)$. Consequently $I_{F}(P ; G, \Omega) \subseteq N_{G, P}\left(V_{F}(P, \Omega)\right)$.

Next let $a \in V_{F}(P, \Omega)$ be a standard basis matrix. As above we calculate $a=$ $N_{P, d}\left(e_{\alpha \beta}\right)$ where $(\alpha, \beta)$ belongs to the orbit of $P$ on $\Omega \times \Omega$ corresponding to $a$ and $d=P_{a \beta}$. Now

$$
N_{G, P}(a)=N_{G, d}\left(e_{\alpha \beta}\right)=N_{G, G_{\alpha \beta}}\left(N_{G_{\alpha \beta}, d}\left(e_{\alpha \beta}\right)\right)=N_{G, G_{\alpha \beta}}\left(\left[G_{a \beta}: d\right] e_{\alpha \beta}\right) .
$$

Thus $N_{G, P}(a)=0$ if $d$ is not a Sylow subgroup of $G_{a \beta}$, and $N_{G, P}(a)$ is a multiple of the standard basis matrix in $V_{F}(G, \Omega)$ corresponding to $(\alpha, \beta)^{G}$ if $d$ is a Sylow subgroup of $G_{\alpha \beta}$. In either case $N_{G, P}(a) \in I_{F}(P ; G, \Omega)$ and so $N_{G, P}\left(V_{F}(P, \Omega)\right) \subseteq$ $I_{F}(P ; G, \Omega)$.

(2.5) Proof of Proposition 2. This follows from (2.4) and the definition of $D(e)$ because of D. Higman's criterion [10, Theorem 1]. See also Green [9, p. 143].

(2.6) Remarks. (a) Note that if $e_{0} \in V_{R}(G, \Omega)$ is a primitive idempotent with $\bar{e}_{0}=e$, then $D(e)$ is also a vertex of $R \Omega e_{0}$ by Proposition 1.

(b) An alternate proof of Lemma 1 can be given by (2.4) and Green's formula $[9,4.11]$.

If $F$ is a splitting field for $V_{F}$ and $e \in V_{F}$ is a primitive idempotent, then there is a unique irreducible modular character $\left({ }^{4}\right) \lambda$ of $V_{F}$ satisfying $\lambda(e)=1$.

(3) We follow Feit's notation [5]. The analogous notation in Green [7] is $T_{G: H}(x)$, and $T_{H, G}(x)$ in Green [9].

(4) Throughout this paper the term "character" refers to the trace function obtained from a module. 
This leads to a second characterization of defect groups.

Proposition 3. Assume $F$ is a splitting field for $V_{F^{*}}$. Let $e, e^{\prime} \in V_{F}$ be primitive idempotents, and let $\lambda, \lambda^{\prime}$ be the associated modular characters. Then

(1) $\lambda\left(x A_{i}\right)=0$ for all $x \in V_{F}$ unless $D_{i} \geq_{G} D(e)$.

(2) $\lambda\left(A_{i}\right) \neq 0$ for some $i$ with $D_{i}={ }_{G} D(e)$.

(3) $e$ is equivalent to $e^{\prime}$ if and only if $D(e)={ }_{G} D\left(e^{\prime}\right)$, and $\lambda\left(A_{i}\right)=\lambda^{\prime}\left(A_{i}\right)$ for all $i$ with $D_{i}={ }_{G} D(e)$.

Before giving the proof we establish a general fact which will be used again in $\$ 6$.

(2.7) Suppose $\lambda$ is the character afforded by an absolutely irreducible representation of a finite dimensional F-algebra $A$, and $e \in A$ is a primitive idempotent with $\lambda(e) \neq 0$. Then the restriction of $\lambda$ to eAe is an algebra bomomorphism.

Proof. Let $\mathcal{L}$ be a matrix representation affording $\lambda$. Then $\mathcal{L}(e)$ is still a primitive idempotent in $\mathfrak{L}(A)$, as is well known. Thus by suitably choosing $\mathfrak{Q}$ we may assume that $\mathscr{L}(e)$ has a 1 in the upper left-hand corner and 0 's everywhere else. Hence for $x \in e A e, \lambda(x)$ is the upper left-hand corner entry of $\mathscr{L}(x)$, and all other entries are 0 . The result is now obvious.

(2.8) Proof of Proposition 3. (1) If $D_{i} \Varangle_{G} D(e)$ then $e \notin I_{F}\left(D_{i} ; G, \Omega\right)$ by defition of $D(e)$. Thus $I_{F}\left(D_{i} ; G, \Omega\right) \cap e V_{F} e$ is contained in the kernel of $\left.\lambda\right|_{e V_{F}} e^{\cdot}$ So $\lambda\left(\operatorname{exA} A_{i}\right)=\lambda\left(\operatorname{ex} A_{i} e\right)=0$ for all $x \in V_{F}$. Clearly the same equation holds if $e$ is replaced by an equivalent idempotent. But an idempotent not equivalent to $e$ is in the kernel of any representation affording $\lambda$. Since $\lambda\left(x A_{i}\right)=\lambda\left(1 x A_{i}\right)$ and 1 is a sum of primitive idempotents, the prof of (1) is complete.

(2) By definition $e$ is an $F$-linear combination of standard basis matrices $A_{i}$ with $D_{i} \leq_{G} D(e)$. Since $\lambda(e) \neq 0$, and $\lambda\left(A_{i}\right)=0$ whenever $D_{i}<_{G} D(e)$ by (1), we must have $\lambda\left(A_{i}\right) \neq 0$ for some $i$ with $D_{i}={ }_{G} D(e)$.

(3) Suppose $D(e)={ }_{G} D\left(e^{\prime}\right)$ and $\lambda\left(A_{i}\right)=\lambda^{\prime}\left(A_{i}\right)$ for all $i$ with $D_{i}={ }_{G} D(e)$. Since $\lambda$ and $\lambda^{\prime}$ vanish on matrices $A_{i}$ with $D_{i}{ }_{G} D(e)$, and $e$ is an $F_{-}$olinear combination of matrices $A_{i}$ with $D_{i} \leq_{G} D(e)$, we have $\lambda^{\prime}(e)=\lambda(e)=1$. Thus $e^{\prime}$ must be equivalent to $e$. The converse is almost trivial.

The following theorem depends on Theorem 3 in $\$ 4$.

(2.9) Theorem 2. A necessary condition that $D$ be the vertex of an indecomposable component of $F \Omega$ is that there exists $a \in \Omega$ and $y \in N_{G}(D)$ such that $D$ is a Sylow p-subgroup of $G_{a a} y^{\text {. }}$

Proof (assuming Theorem 3 (and Proposition 5)). By Theorem 3 and the remark following its proof we can assume $G=N_{G}(P)$. Since any indecomposable component of $F \Omega$ is a component of $F \Gamma$ for some orbit $\Gamma$ of $G$ on $\Omega$, we can assume $G$ is transitive. By Proposition 2 and the definition of defect group, $P$ is a Sylow subgroup of $G_{a \beta}$ for some $\alpha, \beta \in \Omega$. 
3 The Brauer homomorphism. Suppose $P \leq H \leq N_{G}(P)$ where $P$ is a pogroup, and let $\Omega_{P}$ denote the set of fixed points of $P$ in $\Omega$. Then there is a natural homomorphism $f=f_{(G, \Omega, P, H)}$ mapping $V_{F}(G, \Omega)$ into $V_{F}\left(H, \Omega_{P}\right)$ : First define $f$ in the special case $G=H$-if $O_{i} \leq \Omega_{P} \times \Omega_{P}$ define $f\left(A_{i}\right)$ to be the standard basis matrix for. $\left(H, \Omega_{P}\right)$ corresponding to $O_{i}$, and otherwise set $f\left(A_{i}\right)=0$; then extend the definition of $f$ to $V_{F}(H, \Omega)$ by linearity. $f$ is now defined in the general case as the composite of the natural inclusion $V_{F}(G, \Omega) \rightarrow V_{F}(H, \Omega)$ and $f_{(H, \Omega, P, H)^{\circ}}$

We give the verification that $f_{(H, \Omega, P, H)}$ is a homomorphism: Note that $O_{i} \subseteq$ $\Omega_{P} \times \Omega_{P}$ if and only if $P \leq \mathrm{D}_{i}$. Set $J=\Sigma_{P \pm D_{i}} I_{F}\left(D_{i} ; H, \Omega\right)$ and let $B$ denote the set of all $F$-linear combinations of standard basis matrices $A_{i}$ in $V_{F}(H, \Omega)$ satisfying $O_{i} \subseteq \Omega_{P} \times \Omega_{P}$. Then $B$ is an algebra isomorphic to $V_{F}\left(H, \Omega_{P}\right)$ in an obvious way, and $J$ is an ideal in $V_{F}(H, \Omega)$ by Lemma 1. Trivially, $V_{F}(H, \Omega)=$ $J+B$ and $J \cap B=0$. The map $f$ is clearly the composite of the natural projection $V_{F}(H, \Omega) \rightarrow B$ and the obvious isomorphism $B \rightarrow V_{F}\left(H, \Omega_{P}\right)$. Thus $f$ is a homomorphism.

If $A \in V_{F}(G)$ then the restriction $V_{F}(G) \rightarrow V_{F}(H)$ carries $A$ into $J$ if and only if $A=\Sigma_{i=1}^{r} c_{i} A_{i}$ where $O_{i} \cap \Omega_{P} \times \Omega_{P}=\varnothing$-that is, $P \Varangle_{G} D_{i}$-whenever $c_{i} \neq$ 0 . Now the above proof gives

(3.1) The kernel of $f_{(G, \Omega, P, H)}$ is $\Sigma_{P \pm_{G} D_{i}} I_{F}(P ; G, \Omega)$.

The relationship between $f$ and the classical Brauer homomorphism is described below. (See also [15, footnote 8].)

Proposition 4. Assume $C_{G}(P) \leq H$ and let $s: Z(F G) \rightarrow Z(F H)$ be the standard Brauer homomorphism. Then the following diagram is commutative:

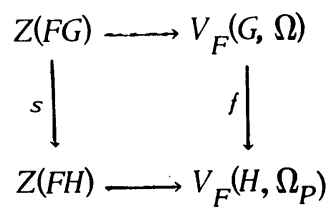

If $C \subseteq G$ we write $\underline{C}$ for the $R$-sum of all permutation matrices corresponding to members of $C$. Thus, if $C$ is a conjugacy class of $G, \underline{C}$ is the image of the class sum $\hat{C} \in Z(R G)$ under the map $Z(R G) \rightarrow V_{R}(G)$. To prove Proposition 4 we need to calculate $\underline{C}$ in terms of standard basis matrices. This has essentially been done by Tamaschke (for the transitive case, in the formalism of Sorings [16]). We give an alternate approach more suitable to the present formalism by using the "orbital character" notation.

For $i=1, \cdots, r$ we define $\theta_{i}(g)=\left|\left\{\alpha \in \Omega \mid\left(\alpha, \alpha^{g}\right) \in O_{i}\right\}\right|$ for each $g \in G$ (as in Scott [14]). Of course $\theta_{i}$ is identically 0 unless $O_{i} \subseteq \alpha^{G} \times \alpha^{G}$ for some $\alpha \in \Omega$.

(3.2) (Scott $[13,2.2]) \theta_{i}(g)$ is the trace of $g A_{i}^{*}$ acting on $R \Omega$.

The proof is a direct calculation from the definition of $A_{i}^{*}$. 
(3.3) $\underline{C}=\Sigma_{i}\left(\left|O_{i}\right|^{-1} \Sigma_{x \in C} \theta_{i}(x)\right) A_{i}$ whenever $C$ is a union of $G$-conjugacy classes.

Proof. Certainly we can express $\underline{C}=\Sigma_{i} c_{i} A_{i}$ for some coefficients $c_{i} \in R$. A particular coefficient $c_{i}$ is computed by multiplying $\underline{C}$ by $A_{i}^{*}$ and taking the trace (the trace of $A_{j} A_{i}^{*}$ on $R \Omega$ is easily calculated to be $\delta_{i j}\left|O_{i}\right|$; see Wielandt $[18, \mathrm{~V}]$ ).

(3.4) (Scott $[13,2.7 .9]$ ) Suppose $C$ is a union of $G$-conjugacy classes. Fix $a$, $\beta \in O_{i}$. Then $\left|O_{i}\right|^{-1} \Sigma_{x \in C} \theta_{i}(x)=\left|\left\{x \in C \mid \alpha^{x}=\beta\right\}\right|$.

Proof. Count the number of triples $(\gamma, \delta, x)$ with $(\gamma, \delta) \in O_{i}, x \in C$, and $\gamma^{x}=$ $\delta$. Counting in terms of $x$ gives a total $\Sigma_{x \in C} \theta_{i}(x)$. Counting in terms of $(\gamma, \delta)$ gives $\left|O_{i}\right|\left|\left\{x \in C \mid \alpha^{x}=\beta\right\}\right|$.

(3.5) Proof of Proposition 4. Fix a conjugacy class $C$ of $G$ and a standard basis matrix $a \in V_{F}\left(H, \Omega_{P}\right)$. Let $\alpha, \beta \in \Omega_{P}$ be such that $(\alpha, \beta)^{H}$ corresponds to a.

Then the $a$-coefficient of $f(\overline{\underline{C}})$ is $\overline{\left|\left\{x \in C \mid a^{x}=\beta\right\}\right|}$ by (3.3) and (3.4). Now $s(\overline{\hat{C}})$ is by definition $\overline{C \cap C_{G}(P)}$. The a-coefficient of $C \cap C_{G}(P)$ is $\overline{\left|\left\{x \in C \cap C_{G}(P) \mid \alpha^{x}=\beta\right\}\right|}$, again by (3.3) and (3.4). Since $P$ fixes $\alpha, \beta$ and centralizes no member of $C-C \cap C_{G}(P)$, we deduce

$$
\left|\left\{x \in C \mid \alpha^{x}=\beta\right\}\right| \equiv\left|\left\{x \in C \cap C_{G}(P) \mid \alpha^{x}=\beta\right\}\right| \quad(p) .
$$

Thus the two $a$-coefficients are equal. Since $C$ and $a$ were arbitrary, the commutativity of the diagram is established.

The following two propositions describe elementary properties of $f(G, \Omega, P, H)$ )

(3.6) Proposition 5. Let $e \in V_{F}(G)$ be a primitive idempotent. Then $f(e) \neq 0$ if and only if $D(e) \geq_{G} P$.

Proof. If $f(e)=0$ then $e \in \Sigma_{P \ddagger G} D_{i} I_{F}\left(D_{i} ; G, \Omega\right)$ by (3.1). Hence $e \epsilon$ $I_{F}\left(D_{i} ; G, \Omega\right)$-consequently $D(e) \leq_{G} D_{i}$-for some $i$ with $D_{i} \Varangle_{G} P$ by Green [7, 3.3a]. Since $P \Varangle_{G} D_{i}$ we have $P \Varangle_{G} D(e)$.

If $f(e) \neq 0$ then $f\left(I_{F}(D(e) ; G, \Omega)\right) \neq 0$, since $e \in I_{F}(D(e) ; G, \Omega)$, and so $P \leq{ }_{G} D_{i}$ by (3.1).

(3.7) Proposition 6. Let $e \in V_{F}(G)$ be a primitive idempotent with $f(e) \neq 0$. If $N$ is an indecomposable component of $F \Omega_{P} f(e)$, then some $D(e)$ contains a vertex of $N$. If $C_{G}(P) \leq H$ then $N$ lies in a block $b$ of $H$ such that $F \Omega e$ is in $b^{G} .(5)$

Proof. It is easy to check that, for any $i, f\left(A_{i}\right)$ is a sum of standard basis matrices with defect group contained in a $G$-conjugate of $D_{i}$. Thus $f(e) \epsilon$ $\Sigma_{Q \leq G} D(e) I_{F}\left(Q ; H, \Omega_{P}\right)$.

(5) See Brauer $[3, \S 2]$ for a definition of $b^{G}$. 
Set $f(e)=\Sigma_{j} e_{j}$ where the $e_{j}$ 's are primitive orthogonal idempotents. Then $N \approx F \Omega_{P} e_{j}$ for some $j$ by the Krull-Schmidt theorem, and the vertex of $N$ is $D\left(e_{j}\right)$ by Proposition 2. Since $f(e) e_{j}=e_{j}$ we have $e_{j} \in \Sigma_{Q \leq G} D(e) I_{F}\left(Q ; H, \Omega_{P}\right)$. So $e_{j} \in I_{F}\left(Q ; H, \Omega_{P}\right)$ for some $Q \leq_{G} D(e)$ by Green $[7,3.3 \mathrm{a}]$. Hence $D\left(e_{j}\right) \leq_{H} Q$ $\leq_{G} D(e)$.

Now suppose $C_{G}(P) \leq H$ so that the standard Brauer homomorphism $s: Z(F G)$ $\rightarrow Z(F H)$ is defined. Let $c \in Z(F G)$ be a primitive idempotent whose image $\underline{c}$ under the map $Z(F G) \rightarrow V_{F}(G)$ satisfies $e \underline{c}=e$ (thus $(F \Omega e) c=F \Omega e$ and so $F \Omega e$ belongs to the block associated with $c)$. Then $f(e)=f(e \underline{c})=f(e) f(\underline{c})=f(e) s(c)$ by Proposition 4. Hence $e_{j}=e_{j} f(e)=e_{j} f(e) s(c)=e_{j} s(c)$; consequently $\left(F \Omega_{P} e_{j}\right) s(c)=F \Omega_{p} e_{j}$. By [3, paragraph following 4.16], the proof is complete.

The following fact is also worth noting.

(3.8) Any indecomposable component of $F \Omega_{P}$ is isomorpbic to a component of $F \Omega_{P} f(e)$ for some primitive idempotent $e \in V_{F}(G)$.

Proof. Since $f(1)=1$, a decomposition $1=\Sigma_{k} e_{k}$ into primitive orthogonal idempotents in $V_{F}(G)$ leads to a decomposition $1=\Sigma_{f\left(e_{k}\right) \neq 0} f\left(e_{k}\right)$ into orthogonal idempotents in $V_{F}\left(H, \Omega_{P}\right)$. The result now follows from the Krull-Schmidt theorem.

At this stage of our development we sacrifice a little simplicity for the sake of obtaining more detailed information.

The 1.1 condition. A pogroup $Q \geq_{G} P$ satisfies the $1-1$ condition with respect to $(G, \Omega, P, H)$ if $P \leq \leq_{G} D_{i}$ whenever $D_{i} \leq_{G} Q$.

The onto condition. A pagroup $Q \geq_{G} P$ satisfies the onto condition with respect to $(G, \Omega, P, H)$ if $o^{G} \cap \Omega_{P} \times \Omega_{P}=o$ whenever $o$ is an orbit of $H$ on $\Omega_{P} \times \Omega_{P}$ whose associated defect group $d$ satisfies $d \leq_{G} Q$.

If $P$ contains no proper subgroup conjugate to any $D_{i}$, then $P$ satisfies the 1-1 condition with respect to $(G, \Omega, P, H)$.

If $H=N_{G}(P)$, and if $P=Q$ (or more generally, if $P$ is weakly closed in $Q$ with respect to $G)$, then $Q$ satisfies the onto condition with respect to $(G, \Omega, P, H)$.

(3.9) We insert here a proof that $Q$ satisfies the onto condition with respect to $(G, \Omega, P, H)$ when $H=N_{G}(P)$ and $P$ is weakly closed in $Q$ with respect to $G$. Let $o$ be an orbit of $H$ on $\Omega_{P} \times \Omega_{P}$ whose associated defect group $d$ is contained in a conjugate of $Q$. Then a straightforward argument shows $P$ is weakly closed in $d$. In particular $N_{G}(d) \leq H$, so $d$ is a full Sylow subgroup of $G_{\alpha \beta}$ for $(\alpha, \beta) \epsilon$ $o$. We now easily deduce that any $G$ conjugate of $P$ contained in $G_{\alpha \beta}$ is $G_{a \beta^{-c o n}-}$ jugate to $P$. By the Jordan-Frattini argument (see [18, proof of 3.5]) $H$ is transitive on the fixed points of $P$ in $o^{G}$. That is, $o^{G} \cap \Omega_{P} \times \Omega_{P}=o$.

If $e$ is any idempotent in $V_{F}(G)$, we let $\Psi_{e}$ denote the character of $G$ afforded by $K \Omega e_{0}$ where $e_{0}$ is an idempotent in $V_{R}(G)$ satisfying $\bar{e}_{0}=e$. In addition we define $\Psi_{e}=0$ for $e=0$. 
Proposition 7. Suppose $Q$ satisfies the 1-1 condition with respect to $(G, \Omega, P, H)$. Let $e, e^{\prime}$ be idempotents in $V_{F}(G)$, and assume that $e$ is primitive with $D(e)={ }_{G} Q$. Then

$$
\left(\Psi_{e}, \Psi_{e^{\prime}}\right)_{G} \leq\left(\Psi_{f(e)}, \Psi_{f\left(e^{\prime}\right)}\right)_{H}
$$

Proposition 8. Suppose $Q$ satisfies the onto condition with respect to $(G, \Omega, P, H)$. Let $e, e^{\prime}$ be idempotents in $V_{F}(G)$, and assume that $e$ is primitive with $D(e)={ }_{G} Q$. Then

$$
\left(\Psi_{e}, \Psi_{e^{\prime}}\right)_{G} \geq\left(\Psi_{f(e)}, \Psi_{f\left(e^{\prime}\right)}\right)_{H}
$$

The following standard fact is needed for the proofs of Propositions 7 and 8.

(3.10) Suppose $e, e^{\prime} \in V_{F}(G)$, and $e^{2}=e,\left(e^{\prime}\right)^{2}=e^{\prime}$. Then $\left(\Psi_{e^{\prime}}, \Psi_{e^{\prime}}\right)=$ $\operatorname{dim}_{F} e V_{F}(G) e^{\prime}$.

Proof. The assertion is trivial in case $e$ or $e^{\prime}$ is 0 ; consequently, we may assume $e, e^{\prime}$ are idempotents. Let $u, u^{\prime}$ be idempotents in $V_{R}(G)$ with $\bar{u}=e$, $\bar{u}^{\prime}=e^{\prime}$. Let $\tilde{K}$ be a splitting field for $V_{K}(G)$. Then obviously $\left(\Psi_{e}, \Psi_{e^{\prime}}\right)=$ $\operatorname{dim}_{\widetilde{K}}\left(\operatorname{Hom}_{\widetilde{K} G}\left(\widetilde{K} \Omega u, \widetilde{K} \Omega u^{\prime}\right)\right)$, and $\operatorname{Hom}_{\widetilde{K} G}\left(\widetilde{K} \Omega u, \widetilde{K} \Omega u^{\prime}\right) \cong u V_{K^{\prime}} u^{\prime}$. Since $V_{\widetilde{K}} \simeq$ $\widetilde{K} \otimes_{R} V_{R}$ and $u V_{R} u^{\prime}$ is an $R$-direct summand of $V_{R}$, we have

$$
\operatorname{dim}_{\widetilde{K}} u V_{\widetilde{K}} u^{\prime}=\operatorname{rank}_{R} u V_{R} u^{\prime}=\operatorname{dim}_{F} \overline{u V_{R} u^{\prime}}=\operatorname{dim}_{F} e V_{F} e^{\prime} \text {. }
$$

(3.11) Proof of Proposition 7. By (3.1) and our hypothesis, the kernel of $f$ intersects the ideal $I_{F}(Q ; G, \Omega)$ trivially. Since $e \in I_{F}(Q ; G, \Omega)$ we have $e V_{F} e^{\prime}$ $\subseteq I_{F}(Q ; G, \Omega)$. Thus $e V_{F} e^{\prime} \approx f\left(e V_{F} e^{\prime}\right) \subseteq f(e) V_{F}\left(H, \Omega_{P}\right) f\left(e^{\prime}\right)$. Application of (3.10) now finishes the proof.

(3.12) Proof of Proposition 8. Set $J=\Sigma_{d \leq G} I_{F}\left(d ; H, \Omega_{P}\right)$. Then $J$ is an ideal in $V_{F}\left(H, \Omega_{P}\right)$, and $J \subseteq f\left(V_{F}(G)\right)$ by hypothesis. By Proposition 6 (or direct calculation) we have $f(e) \in J$. Thus

$$
f(e) V_{F}\left(H, \Omega_{P}\right) f\left(e^{\prime}\right) \subseteq f(e) J f\left(e^{\prime}\right) \subseteq f(e) f\left(V_{F}(G)\right) f\left(e^{\prime}\right) \subseteq f(e) V_{F}\left(H, \Omega_{P}\right) f\left(e^{\prime}\right) .
$$

Therefore $f(e) V_{F}\left(H, \Omega_{P}\right) f\left(e^{\prime}\right)=f(e) f\left(V_{F}(G)\right) f\left(e^{\prime}\right)=f\left(e V_{F}(G) e^{\prime}\right)$. Again application of (3.10) completes the proof.

The next section is devoted to further consequences of the onto condition.

\section{Brauer's first fundamental theorem. We assume throughout this section} that $Q$ satisfies the onto condition with respect to $(G, \Omega, P, H) . P \leq H \leq N_{G}(P)$ and $f=f_{(G, \Omega, P, H)}$ as in $\$ 3$.

(4.1) Theorem 3. (a) Let $e, e^{\prime}$ be idempotents in $V_{F}(G, \Omega)$ with e primitive and $D(e)={ }_{G} Q$. Then $f(e)$ is primitive, and $F \Omega e \mid F \Omega e^{\prime}$ if and only if $F \Omega_{P} f(e) \mid F \Omega_{P} f\left(e^{\prime}\right)$.

(b) In case $H=N_{G}(P)$ and $\tilde{e} \in V_{F}\left(H, \Omega_{P}\right)$ is a primitive idempotent with $D(\tilde{e})=P$, then $\tilde{e}$ is equivalent to $f(e)$ for some primitive $e \in V_{F}(G)$ with $D(e)={ }_{G} P$ 
Proof. (a) We shall use the fact established in (3.12) that $f\left(e V_{F}(G) e^{\prime}\right)=$ $f(e) V_{F}\left(H, \Omega_{P}\right) f\left(e^{\prime}\right)$. Of course we also have $f\left(e^{\prime} V_{F}(G) e\right)=f\left(e^{\prime}\right) V_{F}\left(H, \Omega_{P}\right) f(e)$.

Since $e V_{F}(G) e$ is completely primary, so is $f\left(e V_{F}(G) e\right)=f(e) V_{F}\left(H, \Omega_{P}\right) f(e)$. Hence $f(e)$ is a primitive idempotent. $(f(e) \neq 0$ by Proposition 5.)

If $F \Omega f(e) \mid F \Omega f\left(e^{\prime}\right)$ we have $f(e)=z f\left(e^{\prime}\right) w$ where $z \in f(e) V_{F}\left(H, \Omega_{P}\right) f\left(e^{\prime}\right)$ and $w \in f\left(e^{\prime}\right) V_{F}\left(H, \Omega_{P}\right) f(e)$. Choose $x \in e V_{F}(G) e^{\prime}$ and $y \in e^{\prime} V_{F}(G) e$ with $f(x)=z$ and $f(y)=w$. Then $x e^{\prime} y \in e V_{F}(G) e$, and no power of $x e^{\prime} y$ is 0 since $f\left(x e^{\prime} y\right)=$ $z f\left(e^{\prime}\right) e=f(e)$. Thus $x e^{\prime} y$ is a unit in $e V_{F}(G) e$. Consequently $F \Omega e \mid F \Omega e^{\prime}$.

Conversely if $F \Omega e \mid F \Omega e^{\prime}$, then $e=u e^{\prime} v$ for some $u, v \in V_{F}(G)$. Hence $f(e)=$ $f(u) f\left(e^{\prime}\right) f(v)$ and so $F \Omega f(e) \mid F \Omega f\left(e^{\prime}\right)$.

(b) By the remark following Proposition 6 we have $\tilde{e}=z f(e) w$ for some $z \epsilon$ $\tilde{e} V_{F}\left(H, \Omega_{P}\right), w \in V_{F}\left(H, \Omega_{P}\right) \tilde{e}$ and a primitive idempotent $e \in V_{F}(G)$. Since $\tilde{e}$ belongs to the ideal $I_{F}\left(P ; H, \Omega_{P}\right)$ by hypothesis, we have $z, w \in I_{F}\left(P ; H, \Omega_{P}\right)$.

By Sylow's theorem $P$ is a Sylow $p$-subgroup of $G_{\alpha \beta}$ if and only if $P$ is a Sylow $p$-subgroup of $H_{\alpha \beta}$; when this occurs $H$ is transitive on the nonempty set $(\alpha, \beta)^{G} \cap \Omega_{P} \times \Omega_{P}$. Now we easily deduce that $f\left(I_{F}(P ; G, \Omega)\right)=I_{F}(P ; H, \Omega)$. Hence we can choose $x, y \in I_{F}(P ; G, \Omega)$ such that $z=f(x)$ and $w=f(y)$.

Thus eyxe $\in e V_{F}(G) e \cap I_{F}(P ; G, \Omega)$. Since $f(x e y)=z f(e) w=\tilde{e}$ we see that no power of eyxe is 0 . Consequently eyxe is a unit in the completely primary ring $e V_{F}(G) e$. Therefore $e V_{F}(G) e=e V_{F}(G) e \cap I_{F}(P ; G, \Omega)$, and we conclude that $e \in I_{F}(P ; G, \Omega)$.

By part (a), $f(e)$ is a primitive idempotent. Clearly $f(e)$ is equivalent to $\tilde{e}$.

Since $e . \in I_{F}(P ; G, \Omega)$ we have $D(e) \leq_{G} P$. But $f(e) \neq 0$ so $D(e) \geq_{G} P$ by Proposition 5. Thus $D(e)={ }_{G} P$ and the proof is complete.

The previous theorem and Proposition 5 show that $f$ establishes a 1-1 correspondence between equivalence classes of primitive idempotents in $V_{F}(G, \Omega)$ and $V_{F}\left(N_{G}(P), \Omega_{P}\right)$ with defect group $P$. This correspondence will now be identified with the help of a lemma in the next section.

Proposition 9. Suppose $H=N_{G}(P)$ and $e \in V_{F}(G)$ is a primitive idempotent with $D(e)={ }_{G} P$. Then $F \Omega_{P} f(e)$ is the Green correspondent $\left(^{6}\right)$ of $F \Omega e$. In particular

$$
\Psi_{e}^{(1)} \equiv[G: H] \Psi_{f(e)}(1) \quad\left(p^{\nu_{p}([G: P])+1}\right) .
$$

The following preliminary result is an easy application of Proposition 1; the details are left to the reader.

(4.2) Suppose the RG modules $X, Y$ are direct summands of $R \Omega$. Then $X$ is isomorpbic to a direct summand of $Y$ if and only if $\bar{X}$ is isomorpbic to a direct summand of $\bar{Y}$.

(6) See [8, Theorem 2]. 
Now we use (4.2) to prepare for the proof of Proposition 9.

(4.3) Let $X$ be an $N_{G}(P)$-indecomposable component of $R \Omega_{N_{G}(P)}$, and $Y$ a $G$-indecomposable component of $R \Omega$ with vertex $P$. Then $X$ is the Green correspondent of $Y$ if and only if $\bar{X}$ is the Green correspondent of $\bar{Y}$.

Proof. Of course $\bar{Y}$ is indecomposable with vertex $P$ by Proposition 1 .

If $X$ is the Green correspondent of $Y$, then $X \mid Y_{N_{G}(P)}$ and $X$ has vertex $P$. Thus $\bar{X} \mid \bar{Y}_{N_{G}(P)}$ and $\bar{X}$ is indecomposable with vertex $P$ by Proposition 1 , and so $\bar{X}$ is the Green correspondent of $\bar{Y}$.

Conversely if $\bar{X}$ is the Green correspondent of $\bar{Y}$, then $\bar{X} \mid \bar{Y}_{N_{G}(P)}$ and $\bar{X}$ has vertex $P$. Therefore $X$ has vertex $P$ by Proposition 1 , and $X \mid Y_{N_{G}(P)}$, by (4.2) applied to $N_{G}(P)$. Hence $X$ is the Green correspondent of $Y$.

(4.4) Proof of Proposition 9 (assuming Lemma 2). Lemma 2 gives $F \Omega f(e) \mid(F \Omega e)_{H}$.

Trivially $P \leq D(f(e))$, and $D(f(e)) \leq{ }_{G} P$ by Proposition 6. Thus $D(f(e))={ }_{G} P$, and so $F \Omega_{P} f(e)$ is the Green correspondent of $F \Omega e$.

Now choose primitive idempotents $u, v$ in $V_{R}(G), V_{R}\left(H, \Omega_{P}\right)$ respectively, with $\bar{u}=e$ and $\bar{v}=f(e)$. Thus $\overline{R \Omega u} \cong F \Omega e$ and $\overline{R \Omega_{P} v} \cong F \Omega_{P} f(e)$. By Proposition $1, R \Omega u$ has vertex $P$, and by (4.3) $R \Omega_{p} v$ is the Green correspondent of $R \Omega u$. Thus $\left(R \Omega_{P} v\right)^{G}$ is the direct sum of $R \Omega u$ and indecomposable modules with vertex conjugate to a proper subgroup of $P$. The stated congruence on character degrees now follows from Green [7, Theorem 3].

(4.5) Theorem 4. Assume $F$ is a splitting field for $V_{F}(G, \Omega)$ and $V_{F}\left(H, \Omega_{P}\right)$. Suppose e $\in V_{F}(G)$ is a primitive idempotent with $D(e)={ }_{G} Q$ and let $\lambda, \widetilde{\lambda}$ be the modular chracters associated with $e, f(e)$ respectively. Then $\lambda=\tilde{\lambda} \circ f$.

Proof. Let $\mathcal{L}$ and $\widetilde{\mathscr{L}}$ denote matrix representations affording $\lambda, \widetilde{\lambda}$ respectively. These representations have the same degree by Theorems 1 and 3 . Thus $\mathcal{L}$ and $\tilde{\mathcal{E}} \circ f$ are representations of $V_{F}(G)$ of the same degree. Since $\tilde{\lambda}(f(e))=1$, $\mathcal{L}$ is a constituent of $\widetilde{\mathscr{L}} \circ f$. Thus $\mathscr{L}$ is equivalent to $\widetilde{\mathcal{L}} \circ f$.

5. Brauer's second fundamental theorem. Again, $P \leq H \leq N_{G}(P)$ where $P$ is a $p$-subgroup of $G$, and $f$ denotes $f_{(G, \Omega, P, H)^{\circ}} \theta$ is the permutation character of $G$, and if $B$ is a $p$-block of $G$, we set $\theta_{B}=\Sigma_{\chi \in B}(\theta, \chi) \chi$ (where the $\chi$ 's are absolutely irreducible characters of $G$ ).

Lemma 2. Let $e \in V_{F}(G)$ be an idempotent. Then $(F \Omega e)_{H} \approx F \Omega_{P} f(e) \oplus T$ where $T$ is a summand of $f\left(\Omega-\Omega_{P}\right)$.

(5.1) Before the proof, we clarify the condition on $T$. Suppose $P \triangleleft G$. Let $M$ be an indecomposable component of $F \Omega$. Then $M \mid F \Omega_{P}$ if and only if $P$ is contained in the vertex of $M$. 
Proof. Obviously $P$ is contained in the defect group of every standard basis matrix of $\left(G, \Omega_{P}\right)$ and is contained in the defect group of no standard basis matrix of $\left(G, \Omega-\Omega_{P}\right)$. So the result follows from Proposition 2, the Krull-Schmidt theorem, and the way defect groups for idempotents were defined.

(5.2) Proof of Lemma 2. Obviously we may assume that $G=H$ and $e$ is primitive. By (5.1) and Proposition 5 we have $f(e)=0$ if and only if $F \Omega e \mid F\left(\Omega-\Omega_{P}\right)$. Thus it remains to show $F \Omega_{p} f(e) \approx F \Omega e$ in case $f(e) \neq 0$.

Observe that $f(e)$ is a primitive idempotent since $f$ is onto $\left(f(e) V_{F}\left(H, \Omega_{P}\right) f(e)\right.$ $=f\left(e V_{F}(H, \Omega) e\right)$ is completely primary $)$.

To complete the proof we identify $V_{F}\left(H, \Omega_{P}\right)$ with the algebra $B$ described in the first paragraph of $\$ 3$. The map $f$ is now just the projection $V_{H}(H) \rightarrow B$. In particular $f(f(e))=f(e)$ and so $f(e f(e) e)=f(e) \neq 0$. Consequently ef $(e) e$ is a unit in $e V_{F}(H, \Omega) e$. Therefore $e$ is equivalent to $f(e)$ and the proof is complete.

(5.3) Theorem 5. Suppose $x \in G$ and a power $x^{n}=z$ is a p-element. Let $e \epsilon$ $V_{F}(G)$ be an idempotent, and take $P=\langle z\rangle, x \in H$. Then $\Psi_{e}(x)=\Psi_{f(e)}(x)$. In particular $\Psi_{e}(z)$ is a nonnegative rational integer, and $\left.\mid \Psi_{e} \stackrel{e}{(x)}\right) \mid \leq \Psi_{e}^{(z)}(z) \leq \theta(z)$.

If $e$ is primitive, then $\Psi_{e}(z)>0$ if and only if $z$ is conjugate in $G$ to an element of $D(e)$.

Proof. First we show $\Psi_{e}(x)=\Psi_{f(e)}(x)$. Again we may assume that $G=H$ and $e$ is primitive. By Lemma 2 we need only show $\Psi_{e}(x)=\Psi_{f(e)}(x)=0$ when $F \Omega e \mid F\left(\Omega-\Omega_{P}\right)$. Let $e_{0} \in V_{R}(H)$ be a primitive idempotent with $\bar{e}_{0}=e$. Then $P$ is not contained in the vertex of $R \Omega e_{0}$ by Proposition 1 and (5.1). Therefore, the $p$-part of $x$ is not conjugate to an element of the vertex of $R \Omega e_{0}$, and so $\Psi_{e}(x)=$ 0 by Green [7, Theorem 3]. Of course $\Psi_{f(e)}(x)=0$ since $f(e)=0$ by Proposition 5.

Since $P$ acts trivially on $\Omega_{P}, \Psi_{f(e)}(z)=\Psi_{f(e)}(1) \geq\left|\Psi_{f(e)}(x)\right|$.

$\Psi_{e}(z) \neq 0$ precisely when $\Psi_{f(e)} \neq 0$-that is, $f(e) \neq 0$. By Proposition 5 this occurs if and only if $P$ is conjugate in $G$ to a subgroup of $D(e)$. This completes the proof.

As an immediate consequence of the above theorem (3.8) and Proposition 6, we have

(5.4) Corollary A. Suppose $x \in G$ and a power $x^{n}$ is a p-element. Let $\tilde{\theta}$ be the permutation character for the action of $C_{G}\left(x^{n}\right)$ on the set of fixed points of $x^{n}$ in $\Omega$. Then

$$
\theta_{B}(x)=\sum_{b} \tilde{G}_{=B} \tilde{\theta}_{b}(x) .
$$

In particular, $\theta_{B}\left(x^{n}\right)$ is a nonegative rational integer, and $\left|\theta_{B}(x)\right| \leq \theta_{B}\left(x^{n}\right)$ $\leq \theta\left(x^{n}\right)$. 
6. Defect 0 and 1 . We state all the main results of this section before attempting any proofs.

Assume $K, F$ are splitting fields for $V_{K}, V_{F}$, and let $\chi_{s}, \Psi_{j}$ denote the characters of $G$ afforded by $X_{s}, K M_{j}$ respectively (see $\$ 1$ for notation). Let the symbol $\Sigma_{p} \chi_{s}^{\sigma}$ denote the sum of all distinct $p$-conjugates of $\chi_{s}$ and write $s \sim t$ if $\chi_{s}$ is $p$-conjugate to $\chi_{t}$.

Set $a=\nu_{p}(|G|)$, and define $\nu_{s}=\nu_{p}\left(\chi_{s}(1)\right)$. Put $e=\max \left\{\nu_{p}\left(\left|O_{i}\right|\right)\right\}_{i=1}^{r}$.

By a theorem of Wielandt (see Keller [12]), we have $v_{s} \leq e$ for all $s$. We are interested here in the cases $v_{s}=e$ and $v_{s}=e-1$.

Lemma 3. Suppose the number of $p$-conjugates of $\chi_{s}$ is divisible by $p^{y}$. Then

(a) $y \leq e-v_{s}$.

(b) If $y=e-v_{s}$ then $\chi_{s}$ bas exactly $p^{y} p$-conjugates, and $\Sigma_{p} \chi^{\sigma}=\Psi_{j}$ for some $j$. If $\chi_{s} \subseteq \Psi_{k}$ then $k=j$. The vertex of $M_{j}$ bas order $p^{a-e}$.

Theorem 6. Suppose $v_{s}=e$.

Then $\chi_{s}=\Psi_{j}$ for some $j$. If $\chi_{s} \subseteq \Psi_{k}$ then $k=j$. The vertex of $M_{j}$ bas order $p^{a-e}$.

Also, $\chi_{s}$ is p-rational.

Theorem 7. Suppose $v_{s}=e-1$. Then we bave either $A$ or $B$ below:

(A) $\chi_{s}$ has exactly $p$-conjugates. $\Sigma_{p} \chi_{s}^{\sigma}=\Psi_{j}$ for some $j$, and if $\chi_{s} \subseteq \Psi_{k}$, then $k=j$. The vertex of $M_{j}$ has order $p^{a-e}$.

(B) The number of $p$-conjugates of $\chi_{s}$ divides $p-1$. If $\chi_{s} \subseteq \Psi_{j}$ then one of the following occurs:

(i) $\Psi_{j}=\Sigma_{p} \chi_{s}^{\sigma}$. The vertex of $M_{j}$ bas order $\dot{p}^{a-e+1}$.

(ii) $\Psi_{j}=\Sigma_{p} \chi_{s}^{\sigma}+\Sigma_{p} \chi_{t}^{\sigma}$ where $t \alpha s, v_{t}=e-1$, and the number of $p$-conjugates of $\chi_{t}$ divides $p-1$. The vertex of $M_{j}$ has order $p^{a-e}$. If $\Psi_{j}=\Psi_{k}$, then $k=j$.

(iii) $\Psi_{j}=\Sigma_{p} \chi_{s}^{\sigma}+\Upsilon$ where $\Upsilon \neq 0$ is a character sucb that, whenever $\chi_{t} \subseteq \Upsilon$, the number of $p$-conjugates of $\chi_{t}$ is divisible by $p, v_{t} \leq e-2$, and each $p$-conjugate of $\chi_{t}$ appears in $\Upsilon$ with the same multiplicity as $\chi_{t}$. The vertex of $M_{j}$ bas order $p^{a-e}$. If $\Psi_{j}=\Psi_{k}$, then $k=j$.

(iv) $p=2$ and $\Psi_{j}=2 \chi_{s}$. The vertex of $M_{j}$ bas order $p^{a-e}$. If $\Psi_{j}=\Psi_{k}$, then $k=j$.

Corollary B. Suppose the block $B$ bas defect 1. Let $\langle x\rangle$ be a defect group of $B$, and let $b$ be the block of $N_{G}(\langle x\rangle)$ satisfying $b^{G}=B$. Let $\tilde{\theta}$ be the permutation character of $N_{G}(\langle x\rangle)$ on the fixed points of $x$ in $\Omega$, and set $\tilde{\theta}_{b}=$ $\Sigma_{k} l_{k} \tilde{X}_{k}$, where $\tilde{\chi}_{k}$ is absolutely irreducible and appears in $\tilde{\theta}_{b}$ with multiplicity $l_{k}$. 
Then we may find distinct indices $j_{k}$ such that $\theta_{B}=\Sigma_{k} l_{k} \Psi_{j_{k}}+\Phi$ where

(1) $\Phi$ is the sum (with multiplicities) of characters $\Psi_{j}$ whose affording module $M_{j}$ is projective and lies in $B$.

(2) $\Psi_{j_{k}}(x)>0$ for each $k$, and $\Psi_{j_{k}}$ is either a nonexceptional character of $B$ or the sum of all exceptional characters of $B$.

Now we prepare to give the proofs. Several arithmetic preliminaries are required.

(6.1) There exists a p-adic number field $K$ with the following properties:

(i) $K$ is obtained by adjoining an $m t h$ root of unity, where $p \nmid m$, to the $p$ adic completion of the rational field.

(ii) $K$ contains a primitive lth root of unity, where $l$ is the $p^{\prime}$-part of the group order $|G|$.

(iii) Each character $\chi_{s}$ is afforded by a $K\left(\chi_{s}\right)$ representation.

(iv) Each indecomposable component of $R \Omega$ is absolutely indecomposable (where $R$ denotes the ring of local integers in $K$ ).

Proof. By Fong's result $(7)$ (see $[6,16.5]$ ) a field $K_{0}$ satisfying the first three conditions is obtained by adjoining an $l$ th root of unity (or (3l)th root of unity if $p=2$ and $3 \nmid l)$ to the $p$-adic completion of the rational field. Let $F_{0}$ denote the residue class field of $K_{0}$, and select a finite field $G F(q)$ which is a splitting field for $V_{F_{0}}(G, \Omega)$. Now let $K$ be the field otained by adjoining a primitive $(q-1)$ th root of unity to $K_{0}$. Then the field $K$ satisfies condition (iv) by (1.3). Obviously $K$ satisfies the other three conditions.

(6.2) Notation. Note that it suffices to prove Lemma 3, Theorems 6 and 7 and Corollary B for any particular $p$-adic field satisfying condition (iv) above. Consequently we shall assume throughout the rest of this section that $K$ is a field satisfying all the conditions of (6.1).

We let $\Theta_{s}$ denote the Galois group of $K\left(\chi_{s}\right) / K$, and let $\oint_{s}^{0}$ denote the Sylow $p$-subgroup of $\varrho_{s}$. Set $p^{w_{s}}=\left|\biguplus_{s}^{0}\right|$. $\varrho_{s}$

(6.3) (i) $\Psi_{s}^{\sigma}$ ranges over all the distinct p-conjugates of $\chi_{s}$ as $\sigma$ ranges over

(ii) $\Sigma_{\sigma \epsilon \biguplus_{s}^{0}} y^{\sigma} \equiv 0\left(p^{w_{s}}\right)$ for any local integer $y$ of $K\left(\chi_{s}\right)$.

Proof. By (6.1ii) $\widetilde{Q} \subseteq K$, where $\widetilde{Q}$ is the field of $l$ th roots of unity. Then $K\left(\chi_{s}\right) \subseteq K(\omega)$ where $\omega$ is a primitive $p^{a}$ th root of unity. $K(\omega) / K$ is a fully ramified abelian extension of degree $(p-1) p^{a-1}$ (see [17, proof of 7-4-1]). The $p$-conjugates of $\chi_{s}$ are by $\underset{\sim}{\sim} \sim_{\sim}$ definition the characters $\chi_{s}^{\sigma}, \sigma$ ranging over the Galois group of $\tilde{Q}\left(\chi_{s}\right) / \widetilde{Q}$. Since $\tilde{Q}(\omega) / \tilde{Q}=(p-1) p^{a-1}$, it follows from our description of

(7) Quoting this result to prove (6.1) is, in a historical sense, putting the cart before the horse. The reader familiar with algebras over local fields (see Albert [1, IX, Theorem 23]) can easily prove (6.1) directly. 
$K(\omega) / K$ that $K\left(\chi_{s}\right) / K$ is a fully ramified extension of degree $\left[\tilde{Q}\left(\chi_{s}\right): \tilde{Q}\right]$, and $\mathcal{S}_{s}$ is naturally isomorphic to the Galois group of $\tilde{Q}\left(\chi_{s}\right) / \widetilde{Q}$. In particular (i) has now been proved.

Let $\omega_{s}$ be a primitive $p^{w_{s}+1}$ th root of unity. $K\left(\omega_{s}\right) \subseteq K(\omega)$ since

$$
p^{w_{s}}=\left|\oint_{s}^{0}\right|\left|\left[K\left(\chi_{s}\right): K\right]\right|[K(\omega): K]=(p-1) p^{a-1}
$$

We claim $K\left(\chi_{s}\right) \subseteq K(\omega) .\left({ }^{8}\right)$

Let $\mathcal{E}$ denote the Galois group of $K(\omega) / K$, and let $\mathcal{E}_{\chi}, \mathcal{E}_{\omega}$ be the subgroups of $\mathcal{E}$ which Galois correspond to $K\left(\chi_{s}\right), K\left(\omega_{s}\right)$ respectively. Since $\mathcal{G}_{s}^{0}$ is a Sylow subgroup of $\mathcal{G}_{s}$ and $\mathcal{G}_{s} \cong \mathcal{E}_{/} \mathcal{E}_{x}$, the power of $p$ in $\left|\mathcal{E}_{x}\right|$ is $p^{a-1-w_{s}}$. Since $\mathcal{E} / \mathcal{E}_{\omega}$ is isomorphic to the Galois group of $K\left(\omega_{s}\right) / K$ and $\left[K\left(\omega_{s}\right): K\right]=(p-1) p^{w_{s}}$, we have $\left|\mathcal{E}_{\omega}\right|=p^{a-1-w_{s}}$. Thus $\mathcal{E}_{\omega} \subseteq \mathcal{E}_{x}$ and so $K\left(\omega_{s}\right) \supseteq K\left(\chi_{s}\right)$.

Now let $\mathfrak{S}_{s}^{1}$ denote the group of all automorphisms $\sigma$ of $K\left(\omega_{s}\right) / K$ such that $\omega_{s}^{\sigma}=\omega_{s}^{n}$ for some integer $n \equiv 1(p)$. We claim $\oint_{s}^{1}$ is isomorphic to $\oint_{s}^{0}$ by restriction to $K\left(\chi_{s}\right)$.

Let $\oint_{s}^{2}$ denote the Galois group of $K\left(\omega_{s}\right) / K$. From the definition of $\oint_{s}^{0}$ we have $\left[\varrho_{s}^{2}: \varrho_{s}^{1}\right]=p-1$ and so $\varrho_{s}^{1}$ is a Sylow $p$-subgroup of $\mathcal{G}_{s}^{2}$. The natural restriction map $\varrho_{s}^{2} \rightarrow \varrho_{s}$ is an epimorphism and consequently sends $\varrho_{s}^{1}$ onto $\varrho_{s}^{0}$. But $\left|\varrho_{s}^{1}\right|=p^{w_{s}}=\left|\varrho_{s}^{0}\right|$ and so $\varrho_{s}^{1}$ is isomorphic to $\varrho_{s}^{0}$ by restriction as claimed

Thus to prove (ii) it is enough to show $\Sigma_{\sigma \epsilon \mathcal{S}_{s}^{1}} y^{\sigma} \equiv 0\left(p^{w}\right)$ for any local integer $y$ of $K\left(\omega_{s}\right)$.

Now the powers of $\omega_{s}$ contain an integral basis for $K\left(\omega_{s}\right) / K[17$, proof 7-53]. Thus we need only verify the congruence in case $y=\omega_{s}^{k}$ for some integer $k$. Then

$$
\sum_{\sigma \in \Theta_{s}^{1}} y^{\sigma}=\sum_{z=0}^{p^{w_{s}}-1} \omega_{s}^{(1+z p) k}=\omega_{s}^{k} \sum_{z=0}^{p^{w} s-1}\left(\omega_{s}^{p k}\right)^{z}
$$

The final sum is $p^{w_{s}}$ if $\omega_{s}^{p k}=1$ and $\left(\omega_{s}^{p k}\right) p^{w_{s}}-1 / \omega_{s}^{p k}-1=0$ otherwise. This proves (ii).

(6.4) If $s \sim t$ then $d_{s j}=d_{t j}$ for all $j$.

Proof. By (6.1 iv) each character $\Psi_{j}$ is afforded by an $R G$ representation. Hence $\left(\Psi, \chi_{s}\right)=\left(\Psi_{j}, \chi_{s}^{\sigma}\right)$ for each $\sigma \in \Theta_{s}$ and each $j$. The result now follows from $(6.3 \mathrm{i})$.

(6.5) If $\Psi_{j}=\Psi_{k}$ and $M_{j}, M_{k}$ both bave a vertex of order $p^{a-e}$, then $j=k$.

Proof. Let $P$ be a vertex of $M_{j}$. Then $P$ satisfies both the 1-1 condition and the onto condition with respect to $\left(G, \Omega, P, N_{G}(P)\right)$. Let $e_{j}, e_{k} \in V_{F}(G, \Omega)$

${ }^{(8)}$ This argument fails if $p=2$. But in this case the sum in (ii) is just $\left[\tilde{Q}(\omega): \widetilde{Q}\left(\chi_{s}\right)\right]^{-1} \operatorname{tr} \widetilde{Q}(\omega) / \widetilde{Q}^{(y)}$. 
be primitive idempotents with $\bar{M}_{j} \approx F \Omega e_{j}, \bar{M}_{k} \approx F \Omega e_{k}$. Then Propositions 7 and 8 give

$$
\begin{aligned}
\left(\Psi_{f\left(e_{j}\right)}, \Psi_{f\left(e_{k}\right)}\right) & =\left(\Psi_{e_{j}}, \Psi_{e_{k}}\right)=\left(\Psi_{j}, \Psi_{k}\right) \\
& =\left(\Psi_{j}, \Psi_{j}\right) \quad(\neq 0) \\
& =\left(\Psi_{f\left(e_{j}\right)}, \Psi_{f\left(e_{j}\right)}\right) .
\end{aligned}
$$

Since the inner product is not zero, we have $D\left(e_{k}\right) \geq_{G} P$ by Proposition 5. By hypothesis $\left|D\left(e_{k}\right)\right|=|P|$. Hence $D\left(e_{k}\right)={ }_{G} P$. By symmetry we now have a second equality

$$
\left(\Psi_{f\left(e_{j}\right)}, \Psi_{f\left(e_{k}\right)}\right)=\left(\Psi_{f\left(e_{k}\right)}, \Psi_{f\left(e_{k}\right)}\right)
$$

It follows that $\Psi_{f\left(e_{j}\right)}=\Psi_{f\left(e_{k}\right)}$. Since $F \Omega_{P} f\left(e_{j}\right)$ and $F \Omega_{P} f\left(e_{k}\right)$ are projective indecomposable $F\left(N_{G}(P) / P\right)$ modules (Theorem 3), we deduce that $F \Omega_{P} f\left(e_{j}\right) \approx$ $F \Omega_{p} f\left(e_{k}\right)$ (from Proposition 1 and [4, 7-14 or 8.4.11]). Thus $e_{j}$ is equivalent to $e_{k}$ by Theorem 3 (or Proposition 9) and so $j=k$.

(6.6) Proof of Lemma 3. Select an index $j$ such that $\chi_{s} \subseteq \Psi_{j}$, and choose a

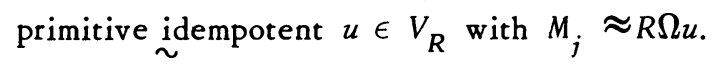

Let $\widetilde{R}$ denote the ring of local integers in $K\left(\chi_{s}\right)=\widetilde{K}$. By $(6.1$ iii) there exists a primitive idempotent $f_{s} \in u V \widetilde{K}^{u}$ such that $\tilde{K} \Omega f_{s}$ affords $\chi_{s}$. By a suitable choice we can insure that $f_{s}$ belongs to an $\widetilde{R}$ order of $u V \widetilde{K}^{u}$ containing $u V \widetilde{R} u$.

Let $\zeta_{s}$ denote the absolutely irreducible character of $V_{\tilde{K}}$ associated with $f_{s}$ (that is, $\left.\zeta_{s}\left(f_{s}\right)=1\right)$. Then $\zeta_{s}\left(f_{s} x\right)=\zeta_{s}\left(u f_{s} u x\right)=\zeta_{s}\left(f_{s} u x u\right) \in \tilde{R}$ for all $x \in V_{\tilde{R}}$, and $\zeta\left(f_{s} x\right)=0$ for any character $\zeta$ of $V \widetilde{K}$ which does not contain $\zeta_{s}$.

We let $\oint_{s}$ act on $V \widetilde{K}$ in the obvious way. For $\sigma \in \mathcal{G}_{s}$ let $\zeta_{s}^{\sigma}$ denote the character associated with $f_{s}^{\sigma}$. Thus $\zeta_{s}^{\sigma}\left(x^{\sigma}\right)=\zeta_{s}(x)^{\sigma}$ for all $x \in V \widetilde{K}^{\text {. }}$.

Since the characters $\chi_{s}^{\sigma}, \sigma \in \mathcal{G}_{s}$, are distinct, the idempotents $f_{s}^{\sigma}$ are orthog-

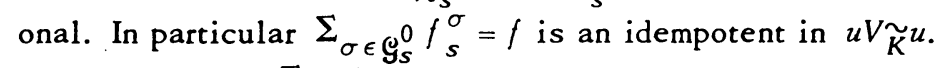

Now set $f=\Sigma t_{i} A_{i}$. We compute the coefficients $t_{i}$ as follows: Let $\tau$ denote the character of $V \widetilde{K}$ afforded by $\tilde{K} \Omega$. Then $\tau=\Sigma_{t} \chi_{t}(1) \zeta_{t}$, and $\tau\left(A_{j} A_{i}^{*}\right)=\delta_{i j}\left|O_{i}\right|$ (as in the proof of (3.3)). Thus $\left|O_{i}\right| t_{i}=r\left(f A_{i}^{*}\right)=\chi_{s}(1) \Sigma_{\sigma \in \varrho_{s}^{0}} \zeta_{s}\left(f A_{i}^{*}\right)^{\sigma}$ for each $i$.

Hence

$$
\begin{aligned}
\nu_{p}\left(t_{i}\right) & =v_{s}+\nu_{p}\left(\sum_{\sigma \in \Theta_{s}^{0}} \zeta_{s}\left(f A_{i}^{*}\right)^{\sigma}\right)-\nu_{p}\left(\left|O_{i}\right|\right) \\
& \geq v_{s}+w_{s}-\nu_{p}\left(\left|O_{i}\right|\right) \text { by }(6.3 \mathrm{ii}) \\
& \geq v_{s}+w_{s}-e .
\end{aligned}
$$


Since $f$ is an idempotent, $\nu_{p}\left(t_{i}\right) \leq 0$ for some $i$. This proves part (a) of Lemma 3.

Now suppose $w_{s}=e-v_{s}$.

Then $\nu_{p}\left(t_{i}\right) \geq 0$ for all $i$ and so $f \in u V \widetilde{R} u$. Hence $f=u$. In particular $\Psi_{j}=$ $\Sigma_{\sigma \in \mathfrak{S}_{s}^{0}} \chi_{s}^{\sigma}$. By (6.4) $\mathfrak{S}_{s}^{0}=\mathfrak{S}_{s}$-that is, $\chi_{s}$ has exactly $p^{w_{s}} p$-conjugates.

If $\nu_{p}\left(t_{i}\right)=0$ then $0 \geq v_{s}+w_{s}-\nu_{p}\left(\left|O_{i}\right|\right) \geq 0$ and so $\nu_{p}\left(\left|O_{i}\right|\right)=v_{s}+w_{s}=e$. So by definition $|D(\bar{u})|=p^{a-e} . D(\bar{u})$ is the vertex of $M_{j}$ by Propositions 1 and 2 .

Since the choice of $j$ was arbitrary except for $\chi_{s} \subseteq \Psi_{j}$, we have $\Psi_{j}=\Psi_{k}=$ $\Sigma_{p} \chi_{s}^{\sigma}$ whenever $\chi_{s} \subseteq \Psi_{k}$. Also the vertex of $M_{k}$ has order $p^{a-e}$; thus $\chi_{s} \subseteq \Psi_{k}$ implies $k=j$ because of (6.5). This completes the proof of part (b) and the lemma.

(6.7) Proof of Theorem 6. Immediate from Lemma 3.

The next result is a technical preliminary to the proof of Theorem 7. Its assertions are almost trivial in case $V_{K}$ is commutative.

(6.8) Suppose $\chi_{s}+\chi_{t} \subseteq \Psi_{j}$ where either $\underset{\sim}{s}=t$ or $s \ngtr t$. Let $u \in V_{R}$ be a primitive idempotent with $R \Omega u \approx M_{j}$, and let $\tilde{K}$ be $\underset{\sim}{\text { a }}$-adic field containing $K\left(\chi_{s}\right), K\left(\chi_{t}\right)$. Denote the ring of local integers in $\widetilde{K}$ by $\widetilde{R}$.

Then there exist primitive idempotents $f_{s}, f_{t} \in V \widetilde{K}$ such that $\tilde{K}_{\Omega} f_{s}$ affords $\chi_{s}, \tilde{K} \Omega f_{t}$ affords $\chi_{t}$, and

(i) $f_{s} \in u V_{K\left(x_{s}\right)} u$ and $f_{i} \in u V_{K\left(x_{t}\right)} u$;

(ii) $f_{s}^{\sigma}$ is orthogonal to $f_{t}^{\tau}$ for each $\sigma \in \mathcal{Y}_{s}$ and $\tau \in \mathcal{S}_{t}$;

(iii) $\zeta_{s}\left(f_{s} w\right) \in \widetilde{R}$ and $\zeta_{t}\left(f_{t} w\right) \in \widetilde{R}$ for all $w \in V_{\tilde{R}}$, where $\zeta_{s}, \zeta_{t}$ are the absolutely irreducible characters of $V_{K}$ satisfying $\zeta_{s}\left(f_{s}\right)=\zeta_{t}\left(f_{t}\right)=1$. Furthermore, $\overline{\zeta_{s}\left(f_{s} w\right)}=\overline{\zeta_{t}\left(f_{t} w\right)} \equiv \overline{(u w u)}$ where $\lambda$ is the absolutely irreducible character of $\overline{V_{\tilde{R}}}$ (with values in $\overline{\widetilde{R}}$ ) satisfying $\lambda(\bar{u})=1$.

Proof. From (the proof of) Theorem 1 we know that $u V_{K\left(x_{s}\right)^{u}}$ has an absolutely irreducible representation module $Y_{s}$ of degree $d_{s j}$ such that $K\left(\chi_{s}\right) \Omega f$ affords $\chi_{s}$ for any primitive idempotent $f \in u V_{K\left(\chi_{s}\right)}^{u}$ with $Y_{s} f \neq 0$. If $\zeta_{s}$ is the character of $V_{K\left(x_{s}\right)}$ associated with $f$ then $\zeta_{s}(f w)$ is easily seen to be the trace of the action of fuwu on $Y_{s}\left(w \in V_{K\left(x_{s}\right)}\right)$. Moreover, if $f_{1}, f_{2}, \cdots$ are a full set of $d_{s j}$ orthogonal primitive idempotents in $u V_{K\left(x_{s}\right)} u$ which do not annihilate $Y_{s}$, then we may view the quantities $\zeta_{s}\left(f_{1} w\right), \zeta_{s}\left(f_{2} w\right), \ldots$ as diagonal coefficients for $u w u$ in a matrix representation associated with $Y_{s}$.

Let $R_{s}$ denote the ring of local integers in $K\left(\chi_{s}\right)$. Now the algebra $\overline{u V_{R_{s}}{ }^{u}}$ has a unique irreducible representation, which is linear and may be realized as the restriction to $\overline{u V_{R_{s}} u}$ of the irreducible character $\lambda$ of $\overline{V_{R_{s}}}$ associated with $\bar{u}$ (see (2.7)). Thus we may choose a matrix representation associated with $Y_{s}$ so that any $x \in u V_{R_{s}} u$ is represented integrally with all diagonal coefficients having residue class $\lambda(x)$. Let $f_{1}, f_{2}, \cdots \in u V_{K\left(x_{s}\right)} u$ be primitive idempotents which give rise to such a matrix representation. 
In case $s=t$ we take $f_{s}=f_{1}, f_{t}=f_{2}$ and it is clear from the preceding discussion that (i) and (iii) are satisfied. (ii) is a consequence of the mutual orthogonality of the idempotents $\left(f_{1}+f_{2}\right)^{\sigma}, \sigma \in \mathcal{G}_{s}$.

In case $s \nsucc t$ we take $f_{s}=f_{1}$ and repeat the whole procedure (with $\chi_{t}$ instead of $\chi_{s}$ ) to obtain $f_{t}$. Here (ii) is automatic, while (i) and (iii) are satisfied as before.

(6.9) Proof of Theorem 7. If the number of $p$-conjugates of $\chi_{s}$ is divisible by $p$, then we have $(A)$ by Lemma 3 . Hence we may assume the number of $p$-conjugates of $\chi_{s}$ is prime to $p$-that is, divides $p-1$.

Suppose $\chi_{s} \subseteq \Psi_{j}$ and let $u \in V_{R}$ be a primitive idempotent with $R \Omega u \approx M_{j}$. We distinguish two main cases.

Case 1. $\Psi_{j}=\Sigma_{p} \chi_{s}^{\sigma}$.

Then $\Psi_{j}(1) \neq 0\left(p^{e}\right)$. Hence by Green [7, Theorem 3] (or Theorem 3) the vertex of $M_{j}$ does not have order $p^{a-e}$. We calculate $u=\Sigma_{i} t_{i} A_{i}$ where $\left|O_{i}\right| t_{i}=$ $\chi_{s}(1) \Sigma_{\sigma \epsilon \Theta_{s}} \zeta_{s}\left(u A_{i}^{*}\right)^{\sigma}$. Hence $\nu_{p}\left(t_{i}\right)>0$ if $\left|D_{i}\right|>p^{a-e+1}$. So by definition $|D(\bar{u})|$ $\leq p^{a-e+1}$. Thus $D(\bar{u})\left(=\right.$ vertex of $\left.M_{j}\right)$ has order exactly $p^{a-e+1}$.

Case 2. $\Psi_{j} \neq \Sigma_{p} \chi_{s}^{\sigma}$.

Here we must show that we get one of the types B (ii), (iii), or (iv). Note that $\Sigma_{p} \chi_{s}^{\sigma} \subseteq \Psi_{j}$ by hypothesis and (6.4). Let $\Sigma_{p} \chi_{s}^{\sigma}+\chi_{t} \subseteq \Psi_{j}$ where $s=t$ or $s+t$.

Set $x_{s}=\chi_{s}(1), x_{t}=\chi_{t}(1)$.

Let $\widetilde{K}$ be a $p$-adic field containing $K\left(\chi_{s}\right), K\left(\chi_{t}\right)$, and choose $f_{s}, f_{t}$ as in (6.8). Then we calculate

$$
\left(p^{e} / x_{s}\right) f_{s}=\sum_{i}\left(p^{e} /\left|O_{i}\right|\right) \zeta_{s}\left(f_{s} A_{i}^{*}\right) A_{i} \in u V_{\widetilde{R}^{u}}^{u}
$$

and

$$
\left(p^{e} / x_{t}\right) f_{t}=\sum_{i}\left(p^{e} /\left|O_{i}\right|\right) \zeta_{t}\left(f_{t} A_{i}^{*}\right) A_{i} \in u V_{\widetilde{R}}^{u}
$$

Thus

$$
\left.\left.\overline{\left(p^{e} / x_{s}\right) f_{s}}=\overline{\left(p^{e} / x_{t}\right) f_{t}}=\sum_{i} \overline{\left(p^{e} /\left|O_{i}\right|\right.}\right) \overline{\left(u A_{i}^{*} u\right.}\right) A_{i} \in \overline{u V_{\tilde{R}} u} .
$$

We show $\overline{\left(p^{e} / x_{s}\right) f_{s}} \neq 0$ : If not, then $\left(p^{e-1} / x_{s}\right) \Sigma_{\sigma \epsilon \varrho_{s}} f_{s}^{\sigma} \in u V_{R} u$ and so $\Sigma_{\sigma \in \varrho_{s}} f_{s}^{\sigma} \in u V_{R} u$. Therefore $\Sigma_{\sigma \in \varrho_{s}} f_{s}^{\sigma}=u$. But this implies $\Psi_{j}=\Sigma_{p} \chi_{s}^{\sigma}$, a contradiction.

In particular $\lambda \overline{\left(u A_{i}^{*} u\right)} \neq 0$ for some $i$ with $\left|D_{i}\right|=p^{a-e}$. By Proposition 3 the vertex of $M_{j}$ has order $p^{a-e}$. Note also that this gives $k=j$ whenever $\Psi_{j}=\Psi_{k}$ because of (6.5).

Suppose now that the number of $p$-conjugates of $\chi_{t}$ divides $p-1$. We will show that this leads to type (ii) or (iv). 
Here we may assume that $\widetilde{K}$ is obtained from $K$ by the adjunction of a primitive pth root of unity (this may be seen directly or from the proof of (6.3)). Set $\mathcal{Y}$ $=\mathscr{G}(\widetilde{K} / K)$ and let $r_{s}=\left|\varrho_{s}\right|, r_{t}=\left|\varrho_{t}\right|$. Write $\hat{f}_{s}$ for $\Sigma_{\sigma \in \Theta_{s}} f_{s}^{\sigma}$ and $\hat{f}_{t}$ for $\Sigma_{\sigma \in \Theta_{t}} f_{t}^{\sigma}$. Then

$$
\left(p-1 / r_{s}\right)\left(p^{\left.e / x_{s}\right)}\right) \hat{f}_{s}-\left(p-1 / r_{t}\right)\left(p^{e} / x_{t}\right) f_{t}=\sum_{\sigma \in \mathcal{S}}\left(\left(p^{e} / x_{s}\right) f_{s}-\left(p^{e} / x_{t}\right) f_{t}\right)^{\sigma} \equiv 0(p) .
$$

Hence $\hat{f}_{s}+c \hat{f}_{t} \in u V_{R} u$ where $c=-r_{s} x_{s} / r_{t} x_{t}$. Now $\nu_{p}\left(x_{t}\right)=v_{t} \leq e-1$ by Theorem 6, and so $c \in R$.

Set $v=\hat{f}_{s}+c \hat{f}_{t}$. Then $\lambda(\bar{v})=\overline{\zeta_{s}\left(f_{s} v\right)}=\overline{\zeta_{s}\left(f_{s}\right)}=1$; also $\lambda(\bar{v})=\overline{\zeta_{t}\left(f_{t} v\right)}=\overline{\zeta_{t}\left(c f_{t}\right)}=$ $\bar{c}$. Therefore $\bar{c}=1$. In particular, $\nu_{t}=\nu_{p}\left(x_{t}\right)=e-1$, and $c=1+\left(p^{e} / x_{t}\right) k$ for some $k \in R$. Now $\hat{f}_{s}+\hat{f}_{t}=v-k\left(p^{e} / x_{t}\right) \hat{f}_{t} \in u V_{R} u$. By (6.8) $\hat{f}_{s}+\hat{f}_{t}$ is an idempotent, and so $\hat{f}_{s}+\hat{f}_{t}=u$. Consequently $\Psi_{j}=\Sigma_{p} \chi_{s}^{\sigma}+\Sigma_{p} \chi_{t}^{\sigma}$. If $s \not t$ we have type (ii). If $s=t$ then $p=2$, since $\bar{c}=1$ and $c=-1$; so we have type (iv).

Finally, we are reduced to the case where the number of $p$-conjugates of $\chi_{t}$ is divisible by $p$ whenever $\Sigma_{p} \chi_{s}^{\sigma}+\chi_{t} \subseteq \Psi_{j}$. By Lemma 3, $v_{t} \leq e-2$. By (6.4) all $p$-conjugates of $\chi_{t}$ appear in $\Psi_{j}$ with the same multiplicity. Thus we have type (iii), and the proof is complete.

(6.10) Proof of Corollary B. Suppose $M_{j}$ is a nonprojective indecomposable component of $R \Omega$ which lies in $B$. Thus the vertex of $M_{j}$ is $\langle x\rangle$ by Green [7, Corollary to Lemma 4.1a]. In particular $\Psi_{j}(x)>0$ by Theorem 5 .

Let $\chi_{s} \subseteq \Psi_{j}$. Then $v_{s}=a-1 \geq e-1$. If $v_{s}=e$ then $\chi_{s}=\Psi_{j}$ is $p$-rational by Theorem 6 . If $v_{s}=e-1$, then $a-e=0$ and so we have type $\mathrm{B}$ (ii), since $M_{j}$ is not projective. Hence $\Psi_{j}=\Sigma_{p} \chi_{s}^{\sigma}$.

Note that the latter case does not occur when $\langle x\rangle$ acts trivially on $\Omega$, because $e$ is obviously less than $a$. So by Theorem 6 the $l_{k}$ 's are the multiplicities of the various indecomposable components of $R \Omega_{x}$ which lie in $b$ ( $R$. suitably large). Application of Theorem 3 now finishes the proof.

\section{REFERENCES}

1. A. A. Albert, Structure of algebras, Amer. Math. Soc. Colloq. Publ., vol. 24, Amer. Math. Soc., Providence, R. I., 1939. MR 1, 99.

2. E. Artin, C. J. Nesbitt and R. M. Thrall, Rings with minimum condition, Univ. of Michigan Publ. in Math., no. 1, Univ. of Michigan Press, Ann Arbor, Mich., 1944. MR 6, 33.

3. R. Brauer, $Z$ ur Darstellungstheorie der Gruppen endlicher Ordnung. II, Math. Z. 72 (1959/60), 25-46. MR $21 \# 7258$.

4. C. W. Curtis and I. Reiner, Representation theory of finite groups and associative algebras, Pure and Appl. Math., vol. 11, Interscience, New York, 1962. MR 26 \#2519.

5. W. Feit, Some properties of the Green correspondence, Theory of Finite Groups (Sympos., Harvard Univ., Cambridge, Mass., 1968), Benjamin, New York, 1969, pp. 139148. MR $39 \# 4295$. 
6. W. Feit, Characters of finite groups, Benjamin, New York, 1967. MR 36 \#2715.

7. J. A. Green, Blocks of modular representations, Math. Z. 79 (1962), 100-115. MR 25 \#5114.

8. - A transfer theorem for modular representations, J. Algebra 1 (1964), 73-84. MR $29 \# 147$.

9. - Some remarks on défect groups, Math. Z. 107 (1968), 133-150. MR 38 \#2222.

10. D. G. Higman, Modules with a group of operators, Duke Math. J 21 (1954), 369376. MR 16, 794.

11. Intersection matrices for finite permutation groups, J. Algebra 6 (1967), 22-42. MR $35 \# 244$.

12. G. Keller, Concerning the degrees of irreducible characters, Math. Z. 107 (1968), 221-224. MR $38 \# 3362$.

13. L. L. Scott, Uniprimitive groups of degree $k p, \mathrm{Ph}$. D. Thesis, Yale University, New Haven, Conn., 1968.

14. Uniprimitive permutation groups, Theory of Finite Groups, (Sympos., Harvard Univ。, Cambridge, Mass., 1968), Benjamin, New York, 1969), pp. 55-62.

15. - The modular theory of permutation representations, Proc. Sympos. Pure Math., vol. 21, Amer. Math. Soc., Providence, R. I. 1971, pp. 137-144.

16. O. Tamaschke, A generalized character theory on finite groups, Proc. Internat. Conf. Theory of Groups, Austral. Nat. U. Canberra, August 1965, New York, 1967, pp. 347355.

17. E. Weiss, Algebraic number theory, McGraw-Hill, New York, 1963. MR 28 \#3021.

18. H. Wielandt, Finite permutation groups, Lectures, University of Tübingen, 1954/55; English transl., Academic Press, New York, 1964. MR 32 \#1252.

DEPARTMENT OF MATHEMATICS, YALE UNIVERSITY, NEW HAVEN CONNECTICUT 06520

Current address: Department of Mathematics, University of Virginia, Charlottesville, Virginia 22904 